\title{
Large-scale distributed L-BFGS
}

\author{
Maryam M. Najafabadi ${ }^{*} \mathbb{B}$, Taghi M. Khoshgoftaar ${ }^{1}$, Flavio Villanustre ${ }^{2}$ and John Holt ${ }^{2}$
}

\section{*Correspondence:}

mmousaarabna2013@fau.edu

${ }^{1}$ Florida Atlantic University,

777 Glades Road, Boca Raton,

FL, USA

Full list of author information is available at the end of the article

\begin{abstract}
With the increasing demand for examining and extracting patterns from massive amounts of data, it is critical to be able to train large models to fulfill the needs that recent advances in the machine learning area create. L-BFGS (Limited-memory Broyden Fletcher Goldfarb Shanno) is a numeric optimization method that has been effectively used for parameter estimation to train various machine learning models. As the number of parameters increase, implementing this algorithm on one single machine can be insufficient, due to the limited number of computational resources available. In this paper, we present a parallelized implementation of the L-BFGS algorithm on a distributed system which includes a cluster of commodity computing machines. We use open source HPCC Systems (High-Performance Computing Cluster) platform as the underlying distributed system to implement the L-BFGS algorithm. We initially provide an overview of the HPCC Systems framework and how it allows for the parallel and distributed computations important for Big Data analytics and, subsequently, we explain our implementation of the L-BFGS algorithm on this platform. Our experimental results show that our large-scale implementation of the L-BFGS algorithm can easily scale from training models with millions of parameters to models with billions of parameters by simply increasing the number of commodity computational nodes.
\end{abstract}

Keywords: Large-scale L-BFGS implementation, Parallel and distributed processing, HPCC systems

\section{Introduction}

A wide range of machine learning algorithms use optimization methods to train the model parameters [1]. In these algorithms, the training phase is formulated as an optimization problem. An objective function, created based on the parameters, needs to be optimized to train the model. An optimization method finds parameter values which minimize the objective function. New advances in the machine learning area, such as deep learning [2], have made the interplay between the optimization methods and machine learning one of the most important aspects of advanced computational science. Optimization methods are proving to be vital in order to train models which are able to extract information and patterns from huge volumes of data.

With the recent interest in Big Data analytics, it is critical to be able to scale machine learning techniques to train large-scale models [3]. In addition, recent breakthroughs in representation learning and deep learning show that large models dramatically improve performance [4]. As the number of model parameters increase, classic implementations of optimization methods on one single machine are no longer feasible. Many 
applications require solving optimization problems with a large number of parameters. Problems of this scale are very common in the Big Data era [5-7]. Therefore, it is important to study the problem of large-scale optimizations on distributed systems.

One of the optimization methods, which is extensively employed in machine learning, is Stochastic gradient descent (SGD) $[8,9]$. SGD is simple to implement and it works fast when the number of training instances is high, as SGD does not use the whole training data in each iteration. However, SGD has its drawbacks, hyper parameters such as learning rate or the convergence criteria need to be tuned manually. If one is not familiar with the application at hand, it can be very difficult to determine a good learning rate or convergence criteria. A standard approach is to train the model with different parameters and test them on a validation dataset. The hyperparameters which give best performance results on the validation dataset are picked. Considering that the search space for SGD hyperparameters can be large, this approach can be computationally expensive and time consuming, especially on large-scale optimizations.

Batch methods such as L-BFGS algorithm, along with the presence of a line search method [10] to automatically find the learning rate, are usually more stable and easier to check for convergence than SGD [11]. L-BFGS uses the approximated second order gradient information which provides a faster convergence toward the minimum. It is a popular algorithm for parameter estimation in machine learning and some works have shown its effectiveness over other optimization algorithms [11-13].

In a large-scale model, the parameters, their gradients, and the L-BFGS historical vectors are too large to fit in the memory of one single computational machine. This also makes the computations too complex to be handled by the processor. Due to this, there is a need for distributed computational platforms which allow parallelized implementations of advanced machine learning algorithms. Consequently, it is important to scale and parallelize L-BFGS effectively in a distributed system to train a large-scale model.

In this paper, we explain a parallelized implementation of the L-BFGS algorithm on HPCC Systems platform. HPCC Systems is an open source, massive parallel-processing computing platform for Big Data processing and analytics [14]. HPCC Systems platform provides a distributed file storage system based on hardware clusters of commodity servers, system software, parallel application processing, and parallel programing development tools in an integrated system.

Another notable existing large-scale tool for distributed implementations is MapReduce [15] and its open source implementation, Hadoop [16]. However, MapReduce was designed for parallel processing and it is ill-suited for the iterative computations inherent in optimization algorithms [4, 17]. HPCC Systems allows for parallelized iterative computations without the need to add any new framework over the current platform and without the limitation of adapting the algorithm to a specific platform (such as MapReduce key-value pairs).

Our approach in implementing L-BFGS over the HPCC Systems platform distributes the parameter vector over many computing nodes. Therefore, a larger number of parameters can be handled by increasing the number of computational nodes. This makes our approach more scalable compared to the typical approaches in parallelized implementations of optimization algorithms, where the global gradient is computed by aggregating the local gradients which are computed on many machines [18]. Each machine maintains 
the whole parameter vector in order to calculate the local gradients on a specific subset of data examples. Thus, handling a larger number of parameters requires increasing the memory on each computational node which makes these approaches harder or even infeasible to scale, where the number of parameters are very large. On the other hand, our approach can scale to handle a very large number of parameters by simply increasing the number of commodity computational nodes (for example by increasing the number of instances on an Amazon Web Services cluster).

The remainder of this paper is organized as follows. In section "Related work", we discuss related work on the topic of distributed implementation of optimization algorithms. Section "HPCC systems platform" explains the HPCC Systems platform and how it provides capabilities for a distributed implementation of the L-BFGS algorithm. Section "L-BFGS algorithm" provides theoretical details of the L-BFGS algorithm. In section "Implementation of L-BFGS on HPCC Systems", we explain our implementation details. In section "Results", we provide our experimental results. Finally, in section "Conclusion and discussion", we conclude our work and provide suggestions for future research.

\section{Related work}

Optimization algorithms are the heart of many modern machine learning algorithms [19]. Some works have explored the scaling of optimization algorithms to build largescale models with numerous parameters through distributed computing and parallelization $[9,18,20,21]$. These methods focus on linear, convex models where global gradients are obtained by adding up the local gradients which are calculated on each computational node. The main limitation of these solutions is that each computational node needs to store the whole parameter vector to be able to calculate the local gradients. This can be infeasible when the number of parameters is very large. In another study, Niu et al. [22] only focus on optimization problems where the gradient is sparse, meaning that most gradient updates only modify small parts of the parameter vector. Such solutions are not general and can only work for a subset of problems.

The research most related to ours are [18] and [4]. Agarwal et al. [18] present a system for learning linear predictors with convex losses on a cluster of 1000 machines. The key component in their system is a communication infrastructure called AllReduce which accumulates and broadcasts values over all nodes. They developed an implementation that is compatible with Hadoop. Each node maintains a local copy of the parameter vector. The L-BFGS algorithm runs locally on each node to accumulate the gradient values locally and the global gradient is obtained by AllReduce. This restricts the parameter vector size to the available memory on only one node. Due to this constraint, their solution only works up to 16 million parameters.

Dean et al. [4] present the Sandblaster batch optimization framework for distributed implementation of L-BFGS. The key idea is to have a centralized sharded parameter server where the parameter vector is stored and manipulated in a distributed manner. To implement distributed L-BFGS, a coordinator process issues commands which are performed independently on each parameter vector shard. Our approach also utilizes a vector partitioning method to store the parameter vector across multiple machines. Unlike [18], where the number of parameters is limited to the available memory on one 
machine, the parameter vector is distributed on many machines which increases the number of parameters that can be stored.

The approach presented in [4] requires a new framework with a parameter server and a coordinator to implement batch optimization algorithms. The approach presented in [18] requires the AllReduce platform on top of MapReduce. However, we do not design or add any new framework on top of the HPCC Systems platform for our implementations. The HPCC Systems platform provides a framework for a general solution for large-scale processing which is not limited to a specific implementation. It allows manipulation of the data locally on each node (similar to the parameter server in [4]). The computational commands are sent to all the computational nodes by a master node (similar to the coordinator approach in [4]). It also allows for aggregating and broadcasting the result globally (similar to AllReduce in [18]). Having all these capabilities, makes the HPCC Systems platform a perfect solution for parallel and large-scale computations. Since it is an open source platform, it allows practitioners to implement parallelized and distributed computations on large amounts of data without the need to design their own specific distributed platform.

\section{HPCC Systems platform HPCC Systems platform}

Parallel relational database technology has proven ineffective in analyzing massive amounts of data [23-25]. As a result, several organizations developed new technologies which utilize large clusters of commodity servers to provide the underlying platform to process and analyze massive data. Some of these technologies include MapReduce [2325], Hadoop [16] and the open source HPCC Systems.

MapReduce is a basic system architecture designed by Google for processing and analyzing large datasets on commodity computing clusters. The MapReduce programming model allows distributed and parallelized transformations and aggregations over a cluster of machines. The Map function converts the input data to groups according to a keyvalue pair, and the Reduce function performs aggregation by key-value on the output of the Map function. For more complex computations, multiple MapReduce calls must be linked in a sequence.

In contrast to MapReduce, in a DataFlow architecture a graph represents a programming unit that performs some kind of transformation on the data. Each node in the graph is an operation. Nodes in a graph are connected by edges representing DataFlow queues. Transferring of the data is done by connecting the DataFlow queues. An example for a DataFlow graph is shown in Fig. 1. First, the data is read from the disk. The next two operations sort and group the data records. Finally some aggregation metrics are extracted from groups of data and the results are written on the disk. This shows how the data is flowing from top to bottom via the connectors of the nodes.

Although MapReduce provides basic functionality for many data processing operations, users are limited since they need to adapt their applications to the MapReduce model to achieve parallelism. This can include the implementation of multiple sequenced operations which can add overhead to the overall processing time. In addition, many processing operations do not naturally fit into the group by-aggregation model using single key-value pairs. Even simple applications such as selection and projection must fit 


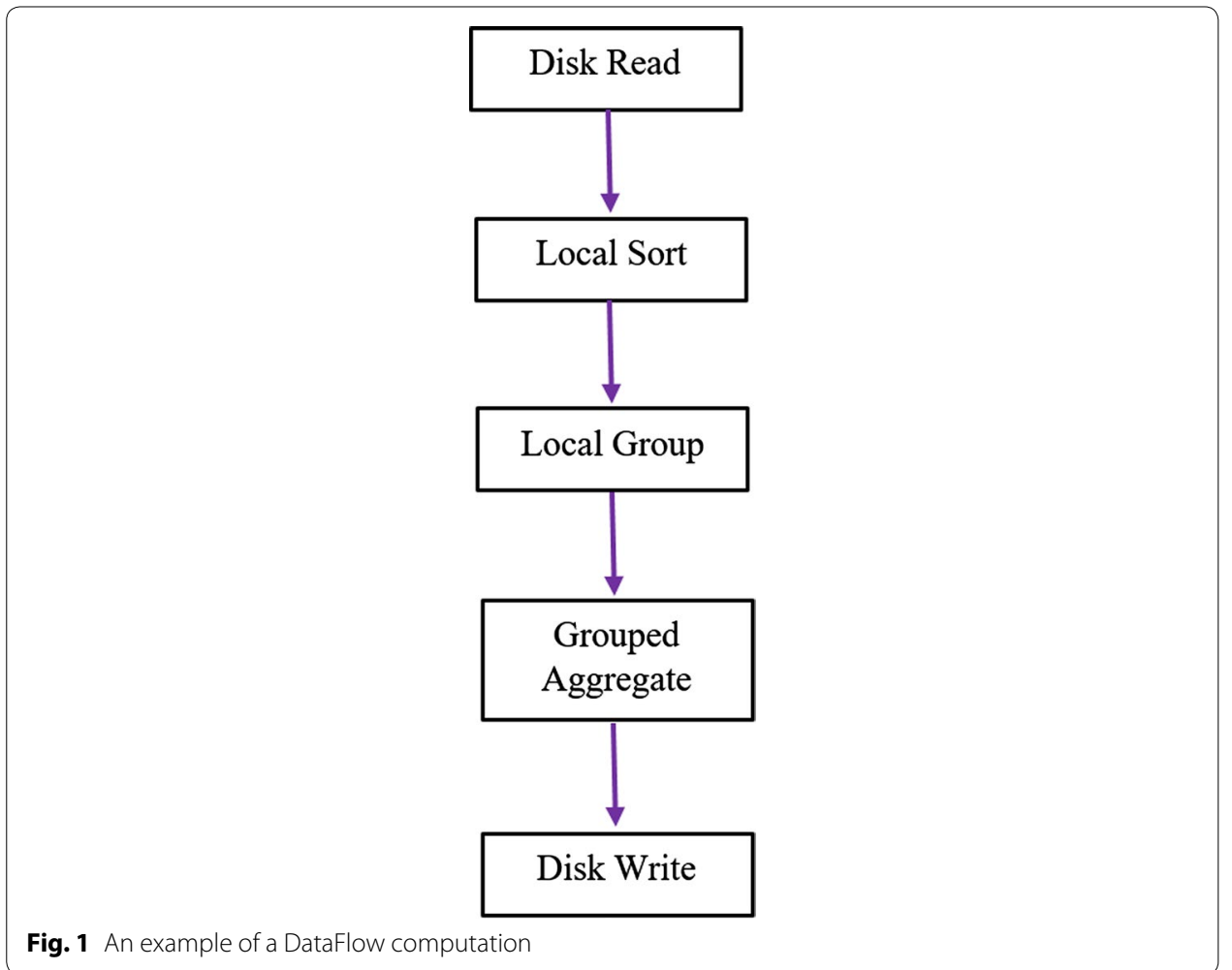

into this model and users need to provide custom MapReduce functions for such operations, which is more error prone and limits re-usability [24].

Some high-level languages, such as Sawzall [26] and Yahoo Pig's system [27], address some of the limitations of the MapReduce model by providing an external DataFloworiented programming language that is eventually translated into MapReduce processing sequences. Even though these languages provide standard data processing operators so users do not have to implement custom Map and Reduce functions, they are externally implemented and not integral to the MapReduce architecture. Thus, they rely on the same infrastructure and limited execution model provided by MapReduce.

HPCC Systems platform, on the other hand, is an open-source integrated system environment which excels at both extract, transform and load (ETL) tasks and complex analytics using a common data centric parallel processing language called Enterprise Control Language (ECL). HPCC Systems platform is based on a DataFlow programming model. LexisNexis Risk Solutions ${ }^{1}$ independently developed and implemented this platform as a solution to large-scale data intensive computing. Similar to Hadoop, the HPCC Systems platform also uses commodity clusters of hardware running on top of the Linux operating system. It also includes additional system software and middleware components to meet the requirements for data-intensive computing such as comprehensive job execution, distributed query and file system support.

\footnotetext{
${ }^{1}$ http://www.lexisnexis.com/.
} 
The data refinery cluster in HPCC Systems (Thor system cluster) is designed for processing massive volumes of raw data which ranges from data cleansing and ETL processing to developing machine learning algorithms and building large-scale models. It functions as a distributed file system with parallel processing power spread across the nodes (machines). A Thor cluster can scale from a single node to thousands of nodes. HPCC also provides another type of cluster, called ROXIE [14], for rapid data delivery which is not in the scope of this paper.

The Thor cluster is implemented using a master/slave topology with a single master and multiple slave processes, which provide a parallel job execution environment for programs coded in ECL. Each slave provides localized data storage and processing power within the distributed file system cluster. The Thor master monitors and coordinates the processing activities of the slave nodes and communicate status information. ECL programs are compiled into optimized $\mathrm{C}++$ source code, which is subsequently linked into executable machine code distributed to the slave processes of a Thor cluster. The distribution of the code is done by the Thor master process. Figure 2 shows a representation of a physical Thor processing cluster.

The distributed file system (DFS) used in the Thor cluster is record oriented which is somewhat different from the block format used in MapReduce clusters. Each record represents one data instance. Records can be fixed or variable length, and support a variety of standard (fixed record size, CSV, XML) and custom formats including nested child datasets. The files are usually transferred to a landing zone and from there they are partitioned and distributed as evenly as possible, with records in sequential order, across the available processes in the cluster.

\section{ECL programming language}

The ECL language is a data-centric, declarative language which allows to define parallel data processing on HPCC Systems. ECL is a flexible language, where its ease of use

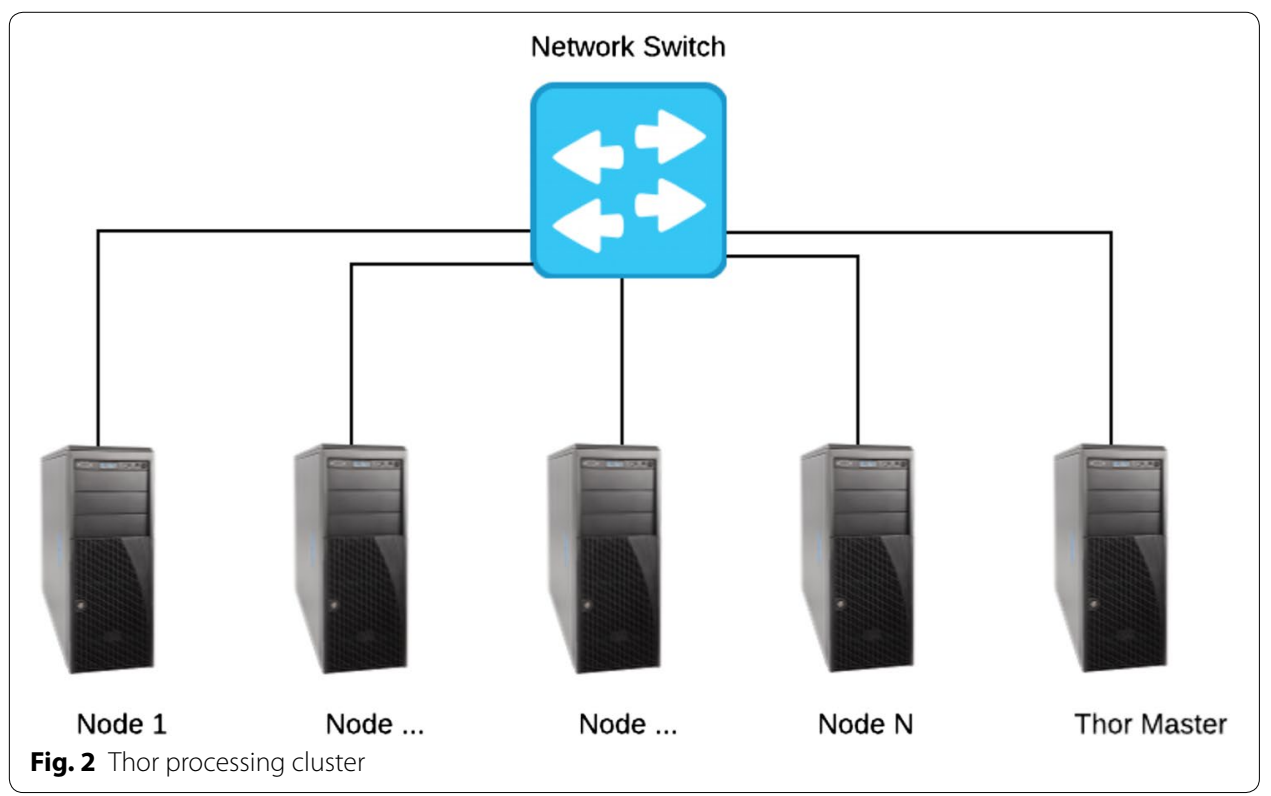


and development speed make HPCC Systems distinguishable from other data-intensive solutions. There are some key benefits with ECL that can be summarized as follows [14]:

- Enterprise Control Language incorporates transparent and implicit data parallelism regardless of the size of the computing clusters reducing the complexity of the parallel programming.

- Enterprise Control Language was specifically designed for manipulation of large amounts of data. It enables implementation of data intensive applications with complex data-flows and huge volumes of data.

- Since ECL is a higher-level abstraction over $\mathrm{C}++$, it provides more productivity improvements for programmers over languages such as Java and $\mathrm{C}++$. The ECL compiler generates highly optimized $\mathrm{C}++$ for execution.

The ECL programming language is a key factor in the flexibility and capabilities of the HPCC Systems processing environment. ECL is designed following the DataFlow model with the purpose of being transparent and an implicit parallel programming language for data-intensive applications. It is a declarative language which allows the programmer to define the data flow between operations and the DataFlow transformations that are necessary to achieve the results. In a declarative language, the execution is not determined by the order of the language statements, but from the sequence of the operations and transformations in a DataFlow represented by the data dependencies. This is very similar to the declarative style recently introduced by Google TensorFlow [28].

DataFlows defined in ECL are parallelized across the slave nodes which process partitions of the data. ECL includes extensive capabilities for data definition, filtering and data transformations. ECL is compiled into optimized $\mathrm{C}++$ format and it allows in-line $\mathrm{C}++$ functions to be incorporated into ECL statements. This allows the general data transformation and flow to be represented with ECL code, while the more complex internal manipulations on data records can be implemented as in-line $\mathrm{C}++$ functions. This makes the ECL language distinguishable from other programing languages for datacentric implementations.

Enterprise Control Language transform functions operate on a single record or pair of records at a time depending on the operations. Built-in transform operations in the ECL language which process through entire datasets include PROJECT, ITERATE, ROLLUP, JOIN, COMBINE, FETCH, NORMALIZE, DENORMALIZE, and PROCESS. For example, the transformation function for the JOIN operation, receives two records at a time and performs the join operation on them. The join operation can be as simple as finding the minimum of two values or as complex as a complicated user-defined in-line $\mathrm{C}++$ function.

The Thor system allows data transformation operations to be performed either locally on each physical node or globally across all nodes. For example, a global maximum can be found by aggregating all the local maximums obtained on each node. This is similar to the MapReduce approach, however, the big advantage of ECL is that this is done naturally and there is no need to define any key-value pair or any Map or Reduce functions. 


\section{L-BFGS algorithm}

Most of the optimization methods start with an initial guess for $x$ in order to minimize an objective function $f(x)$. They iteratively generate a sequence of improving approximate solutions for $x$ until a termination criteria is satisfied. In each iteration, the algorithm finds a direction $p_{k}$ and moves along this direction from the current iterate $x_{k}$ to a new iterate $x_{k+1}$ that has a lower function value. The distance $\alpha_{k}$ to move along $p_{k}$ can be a constant value provided as a hyper-parameter to the optimization algorithm (e.g. SGD algorithm) or it can be calculated using a line search method (e.g. L-BFGS algorithm). The iteration is given by:

$$
x_{k+1}=x_{k}+\alpha_{k} p_{k}
$$

where $x_{k}$ is the current point and $x_{k+1}$ is the new/updated point. Based on the terminology provided in [10], $p_{k}$ is called the step direction and $\alpha_{k}$ is called the step length. Different optimization methods calculate these two values differently. Newton optimization methods use the second order gradient information to calculate the step direction. This includes calculating the inverse of the Hessian matrix. In a high dimensional setting, where the parameter vector $x$ is very large, the calculation of the inverse of a Hessian matrix can get too expensive to compute. Quasi Newton methods overcome the problem of calculating the inverse of Hessian matrix in each iteration, by continuously updating an approximation of the inverse of the Hessian matrix in each iteration.

The most popular Quasi Newton algorithm is the BFGS method, named for its discoverers, Broyden, Fletcher, Goldfarb, and Shanno [10]. In this method, $\alpha_{k}$ is chosen to satisfy the Wolfe Condition [29] so there is no need to manually select a constant value for $\alpha_{k}$. The step direction $p_{k}$ is calculated based on an approximation of the inverse of the Hessian matrix. In each iteration, the approximation of the inverse of the Hessian matrix is updated based on the current $s_{k}$ and $y_{k}$ values. Where $s_{k}$ presents the position difference and $y_{k}$ represents the gradient difference in the iteration. These vectors are the same length as vector $x$.

$$
\begin{aligned}
& s_{k}=x_{k+1}-x_{k} \\
& y_{k}=\Delta f_{k+1}-\Delta f_{k}
\end{aligned}
$$

BFGS needs to keep an approximation of the inverse of the Hessian matrix in each iteration (an $n \times n$ matrix), where $n$ is the length of the parameter vector $x$. It becomes infeasible to store this matrix in the memory for large values of $n$.

The L-BFGS (Limited-memory BFGS) algorithm modifies BFGS to obtain Hessian approximations that can be stored in just a few vectors of the length $n$. Instead of storing a fully dense $n \times n$ approximation, L-BFGS stores just $m$ vectors $(m \ll n)$ of length $n$ that implicitly represent the approximation. The main idea is that it uses curvature information from the most recent iterations. The curvature information from earlier iterations are considered to be less likely to be relevant to the Hessian behavior at the current iteration and are discarded in the favor of the memory.

In L-BFGS, the $\left\{s_{k}, y_{k}\right\}$ pairs are stored from the last $m$ iteration which causes the algorithm to need $2 \times m \times n$ storage compared to $n \times n$ storage in the BFGS algorithm. The $2 \times m$ memory vectors, along with the gradient at the current point, are used in the 
L-BFGS two-loop recursion algorithm to calculate the step direction. The L-BFGS algorithm and its two-loop recursion are shown in Algorithms 1 and 2, respectively. The next section covers our implementation of this algorithm on HPCC Systems platform.
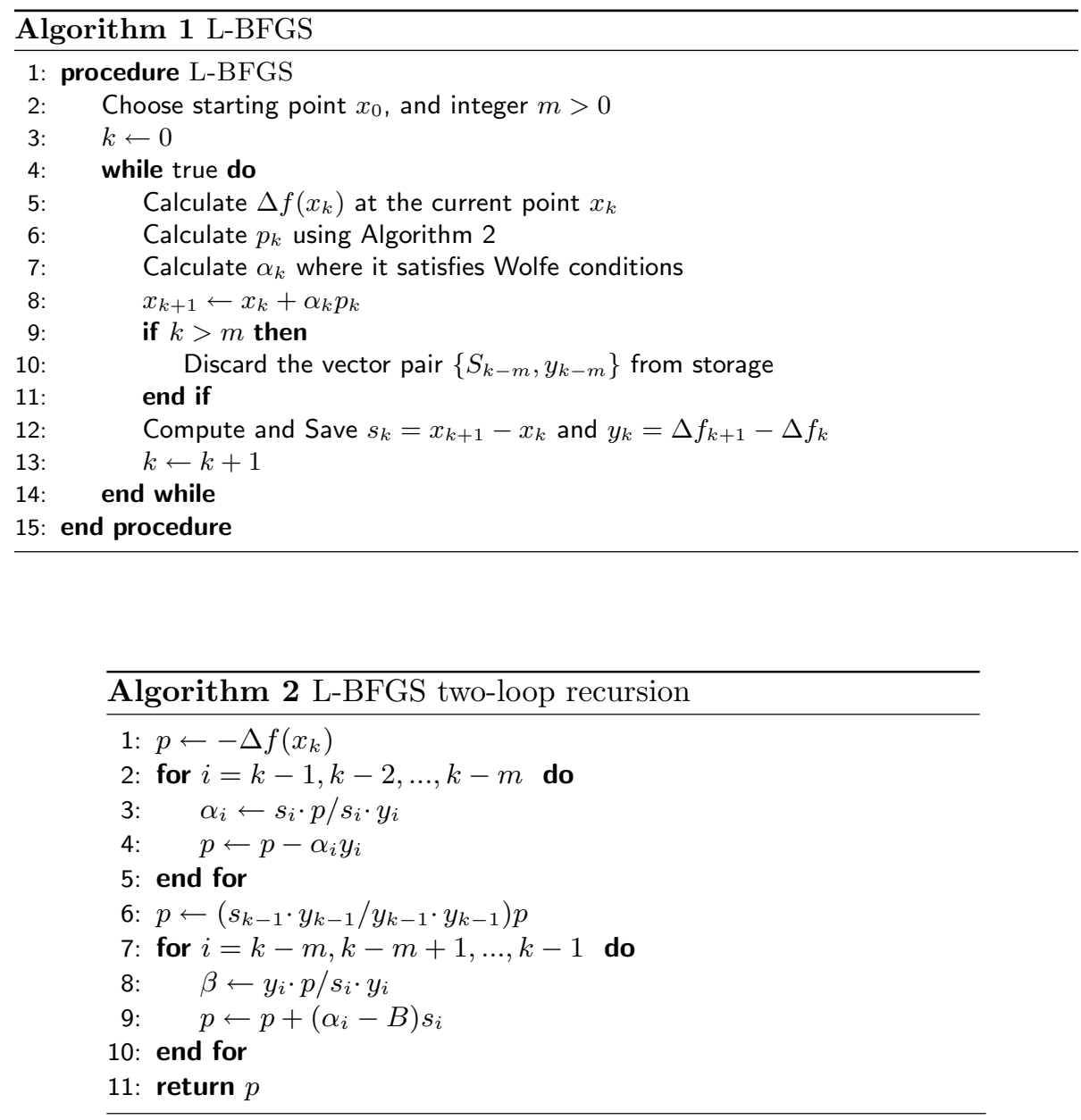

\section{Implementation of L-BFGS on HPCC Systems}

\section{Main idea}

We used the ECL language to implement the main DataFlow in the L-BFGS algorithm. We also implemented in-line $\mathrm{C}++$ functions as required to perform some local computations. We used the HPCC Systems platform without adding any new framework on top of it or modifying any underlying platform configuration.

To implement a large-scale L-BFGS algorithm where the length of the parameter vector $x$ is very large, a natural solution would be to store and manipulate the vector $x$ on several computing machines/nodes. If we use $N$ machines, the parameter vector is divided into $N$ non-overlapping partitions. Each partition is stored and manipulated locally on each machine. For example, if there are 100 machines available and the length of the vector $x$ is $8^{10}(80 \mathrm{~GB})$, each machine ends up storing $\frac{1}{100}$ of the parameter vector which requires $0.8 \mathrm{~GB}$ of memory. By using this approach, the problem of handling a 
parameter vector of size $80 \mathrm{~GB}$ is broken down to handling only $0.8 \mathrm{~GB}$ of partial vectors locally across 100 computational nodes. Even a machine with enough memory to store such a large parameter vector will need even more memory for the intermediate computations and will take a significant amount of time to run only one iteration. Distributing the storage and computations on several machines benefits both memory requirements and computational durations.

The main idea in the implementation of our paralellized L-BFGS alorithm is to distribute the parameter vector over many machines. Each machine manipulates the portion of the locally assigned parameter vector. The L-BFGS caches $\left(\left\{s_{i}, y_{i}\right\}\right.$ pairs) are also stored on the machines locally. For example, if the $j$ th machine stores the $j$ th partition of the parameter vector, it also ends up storing the $j$ th partition of the $s_{i}$ and $y_{i}$ vectors by performing all the computations locally. Each machine performs most of the operations independently. For instance, the summation of two vectors that are both distributed on several machines, includes adding up their corresponding partitions on each machine locally.

By looking at the L-BFGS algorithm two-loop recursion shown in Algorithm 2, it is clear that all the computations can be performed locally, except the dot product calculation. As mentioned earlier, each machine stores a partition of the parameter vector and all the corresponding partitions of the $\left\{s_{i}, y_{i}\right\}$ pairs. Therefore, we calculate the partial dot products on each machine locally. We then add up the local dot product results globally to obtain the final dot product result. Figure 3 shows the dot product computation. In the ECL language, a result which is computed by a global aggregation will be automatically accessible on all the nodes locally. There is no need to include any explicit ECL statement in the code to broadcast the global result on all the nodes.

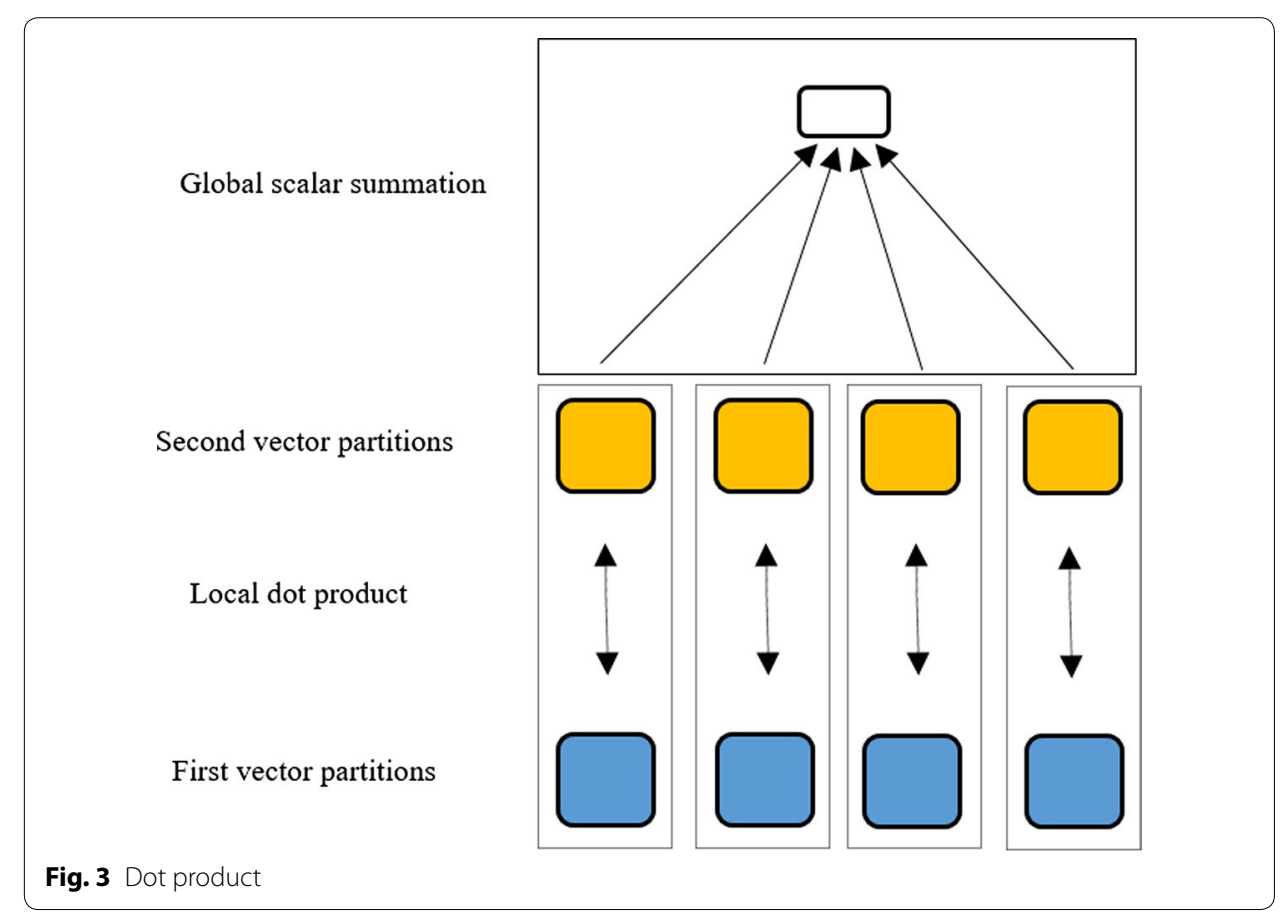




\section{ECL implementation}

In this subsection, we explain our implementation using ECL language by providing some examples from the code. The goal is to demonstrate the simplicity of the ECL language as a language which provides parallelized computations. We refer the interested reader to ECL manual [30] for a detailed explanation of the ECL language.

As mentioned in "HPCC Systems platform", the distributed file system (DFS) used in a Thor cluster is record oriented. Therefore, we represent each partition of the parameter vector $x$ as a single record. Each record is stored locally on each computational node. Each record consists of several fields. For example, one field represents the node id on which the record is stored. Another field includes the partition values as a set of real numbers. The record definition in ECL language is shown below. It should be noted that since ECL is a declarative language, the $:=$ sign implies the declaration and should be read "is defined as".

$\mathrm{x}$ record $:=\mathrm{RECORD}$

UNSIGNED node_id;

SET OF REAL partition_values;

END;

The record can include other fields as required by the computations. For simplicity, we only show the records related to the actual data and its distribution over several nodes.

The aggregation of the records stored in all the nodes builds a dataset which represents the vector $x$.

$\mathrm{x}:=\operatorname{DATASET}(\ldots, \mathrm{x}$ record $)$;

The above statement defines vector $x$ as a dataset of records where each record has the $\mathrm{x} \_$record format. The "..." includes the actual parameter vector $x$ values which can be a file that contains the initial parameter values or it can be a predefined dataset which is defined in ECL. We exclude that part for simplicity.

Distributing this dataset over several machines is as easy as using a DISTRIBUTE statement in ECL and providing the node_id field as the distribution key. Since we only

x_distributed := DISTRIBUTE $(\mathrm{x}$, node_id);

have one record per node_id, this means each record is stored on one machine.

At this point, our initial parameter vector $x$ is represented by a dataset called x_distributed where each record represents one partition and it is distributed on one individual node. The ECL language allows for the computational operations to be done on the dataset records in form of PROJECTs, JOINs, ROLLUPs, etc, locally or globally. For example, scaling the vector $x$ by a constant number can be done as a PROJECT operation. The PROJECT operation processes through all records in the record set and performs a TRANFORM function on each record in turn. The TRANFORM function can be defined to multiply each vector partition by a constant number on each machine locally. 
Table 1 Dataset characteristics

\begin{tabular}{lcccrl}
\hline Dataset & \# Instances & \# Classes & \# Features & \# Parameters & Parameter size (GB) \\
\hline Ishtc-small & 4463 & 1139 & 51,033 & $58,126,587$ & 0.5 \\
Ishtc-large & 93,805 & 12,294 & 347,256 & $4,269,165,264$ & 34 \\
Wikipedia-medium & 456,886 & 31,521 & 346,299 & $10,915,690,779$ & 87 \\
\hline
\end{tabular}

x_distributed_scaled := PROJECT (x_distributed, TRANSFORM(...), LO-

CAL);

The LOCAL keyword specifies the operation is performed on each computational node independently, without requiring interaction with all other nodes to acquire data. The TRANSFORM (...) defines the type of transformation that should be done on each record. In this case, the SET of real values in the "partition_values" field of each record is multiplied by a constant value and the "node_id" field remains the same. The local computations result in the corresponding records from the initial and the result datasets to end up on the same node. All the local computations in our implementation of L-BFGS algorithm are performed in the same manner. This results in the corresponding partitions from the L-BFGS cache information vectors, $\left\{s_{k}, y_{k}\right\}$ pairs, the parameter vector itself and its gradient to end up on the same node with the same node_id values, which is important for a JOIN operation as shown below in the dot product operation. The dot product operation can be done using the JOIN statement.

x_y_dotProduct := JOIN (x_distributed, y_distributed, LEFT.node_id = RIGHT.node id, TRANSFORM(...), LOCAL);

The above statement is joining the two datasets $\mathrm{x}$ _distributed and $\mathrm{y}$ _distributed where both datasets are distributed over several machines. The JOIN operation is performed locally by pairing the records from the left dataset (x_distributed) and the right dataset (y_distributed) with the same node_id values. The LOCAL keyword results in the two records to be joined locally. The transform function returns the local dot product value for each node_id. Using a simple SUM statement provides the final dot product result. The dot product result can then be used in any operation without any explicit reference to the fact that this is a global value that needs to broadcast to local machines. The HPCC Systems platform implicitly broadcasts such global values on local machines.

\section{Results}

To showcase the effectiveness of our implementation, we consider three different datasets with increasing number of parameters, lshtc-small, lshtc-large and wikipediamedium which are large-scale text classification datasets. ${ }^{2}$ The characteristics of the datasets are shown in Table 1. Each instance in the wikipedia-medium dataset can

\footnotetext{
${ }^{2}$ http://lshtc.iit.demokritos.gr/.
} 

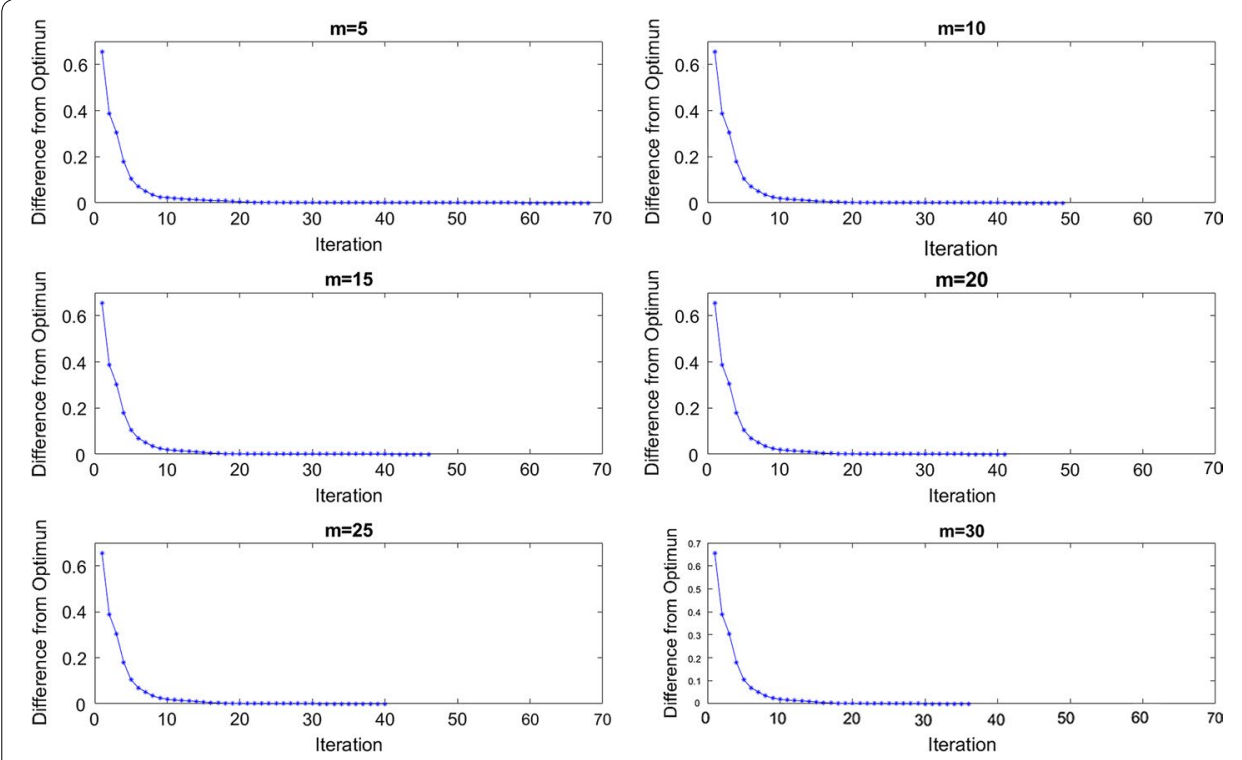

Fig. 4 L-BFGS convergence on Ishtc-small dataset $(\lambda=0.0001)$

belong to more than one class. To build the SoftMax objective function, we only considered the very first class among the multiple classes listed for each sample as its label for the wikipedia-medium dataset.

We used the implemented L-BFGS algorithm to optimize the Softmax regression objective function [31] for these datasets. Softmax regression (or multinomial logistic regression) is a generalization of logistic regression for the case where there are multiple classes to be classified. The number of parameters for the SoftMax regression is equal to the multiplication of the number of classes by the number of features. We use double precision to represent real numbers ( 8 bytes). The parameter size column in Table 1 approximates the memory size which is needed to store the parameter vector by multiplying the number of parameters by 8 . Since the parameter vector is not sparse, we store it as a dense vector which include continuous real values.

We used a cluster of 20 machines, each with 4GB of RAM memory for the lshtc-small dataset. We used an AWS (Amazon Web Service) cluster with 16 instances of r3.8xlarge ${ }^{3}$ (each instance runs 25 THOR nodes) for lshtc-large and wikipedia-medium datasets where each node has almost 9GB of RAM.

Figure 4 and 5 show the difference from the optimal solution as the number of iterations increases for different values of $m$ in the L-BFGS algorithm for lshtc-small and lshtc-large datasets, respectively. We chose the regularization parameter as $\lambda=0.0001$ for these two datasets. We chose a $\lambda$ value that causes the L-BFGS algorithm not to converge as quickly so we can demonstrate more iterations in our results. Figure 6 shows the difference from the optimal solution as the number of iterations increases for $m=5$ for wikipedia-medium dataset. For this dataset, we chose $\lambda=0.0001$ in addition to $\lambda=0.0001$ because the L-BFGS algorithm converges very fast in the case of $\lambda=0.0001$.

${ }_{3}$ https://aws.amazon.com/ec2/instance-types/ 

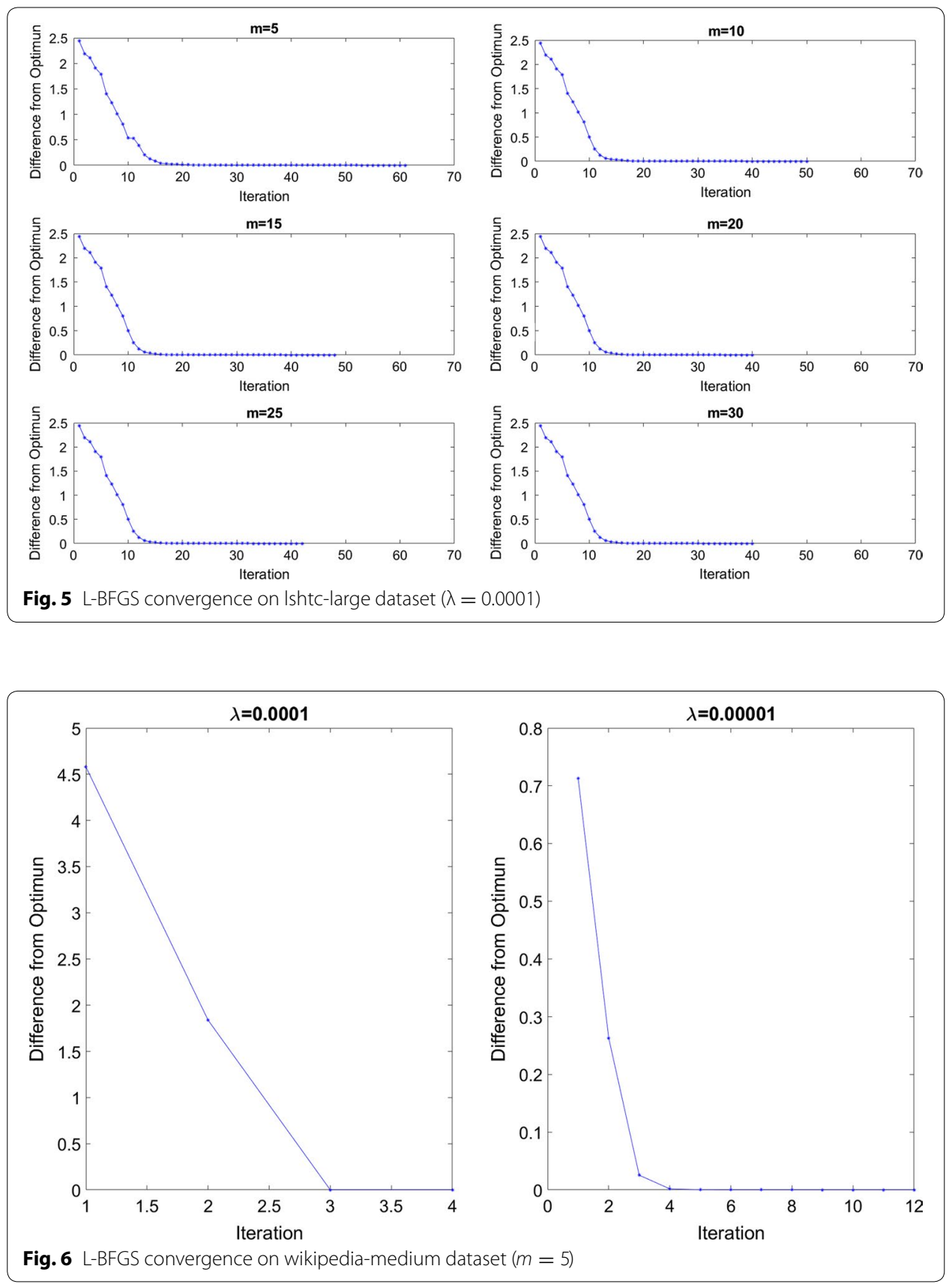

The reason we only considered $m=5$ for this datset is that the number of iteration for the L-BFGS algorithm is small.

Tables 2, 3, and 4 present the corresponding information for the results shown in Figs. 4, 5, and 6, respectively. The number of iterations is the value where the L-BFGS algorithm reached the optimum point. We define the ending criteria for our L-BFGS algorithm the same as the ending criteria defined in minfunc library [32]. Since in each iteration, the Wolfe line search might need to calculate the objective function more than once to find the best step length, the overall number of times the objective function is 
Table 2 Results description for Ishtc-small dataset

\begin{tabular}{lllll}
\hline $\mathbf{m}$ & Memory (GB) & \# Iterations & \# Objective function & Duration (s) \\
\hline 5 & 5 & 68 & 77 & 652 \\
10 & 10 & 49 & 58 & 603 \\
15 & 15 & 46 & 54 & 668 \\
20 & 20 & 41 & 48 & 655 \\
25 & 24 & 40 & 46 & 686 \\
30 & 29 & 36 & 44 & 641 \\
\hline
\end{tabular}

Table 3 Results description for Ishtc-large dataset

\begin{tabular}{lllll}
\hline $\mathbf{m}$ & Memory (GB) & \# Iterations & \# Objective function & Duration (s) \\
\hline 5 & 410 & 61 & 83 & 3752 \\
10 & 751 & 50 & 69 & 3371 \\
15 & 1093 & 48 & 64 & 3360 \\
20 & 1434 & 57 & 40 & 2869 \\
25 & 1776 & 58 & 42 & 3076 \\
30 & 2117 & 57 & 40 & 3012 \\
\hline
\end{tabular}

Table 4 Results description for wikipedia-medium dataset

\begin{tabular}{llclc}
\hline $\boldsymbol{\lambda}$ & Memory (GB) & \# Iterations & \# Objective function & Duration (s) \\
\hline 0.0001 & 1048 & 4 & 11 & 202 \\
0.00001 & 1048 & 12 & 21 & 1297 \\
\hline
\end{tabular}

calculated is usually more than the number of iterations in the L-BFGS algorithm. The total memory usage in these tables presents the required memory by the L-BFGS algorithm. It includes the memory required to store the updated parameter vector, the gradient vector, and $2 \times m$ L-BFGS cache vectors.

The results indicate that increasing the $m$ value in the L-BFGS algorithm causes the algorithm to reach the optimum point in less number of iterations. However, the time it takes for the algorithm to reach the optimum point does not necessarily decrease. The reason is that increasing $m$ causes the calculation of step direction in L-BFGS two-loop recursion algorithm takes more time.

Our results show that the implemented L-BFGS algorithm on the HPCC Systems can easily scale from handling millions of parameters on dozens of computational nodes to handling billions of parameters on hundreds of machines in a reasonable amount of time. Handling the lshtc-small dataset on 20 computational nodes takes less than 15 min. The lshtc-large dataset with more than 4 billion parameters and the wikipediamedium dataset with more than 10 billion parameters on a cluster of 400 nodes takes almost $1 \mathrm{~h}$ and half an hour, respectively. Although, wikipedia-medium is a larger dataset compared to lshtc-large dataset, the L-BFGS algorithm converges in a shorter time, because it requires a smaller number of iterations to reach the optimum. 


\section{Conclusion and discussion}

In this paper, we explained a parallelized distributed implementation of L-BFGS which works for training large-scale models with billions of parameters. The L-BFGS algorithm is an effective parameter optimization method which can be used for parameter estimation for various machine learning problems. We implemented the L-BFGS algorithm on HPCC Systems which is an open source, data-intensive computing system platform originally developed by LexisNexis Risk Solutions. Our main idea to implement the L-BFGS algorithm for large-scale models, where the number of parameters is very large, is to divide the parameter vector into partitions. Each partition is stored and manipulated locally on one computational node. In the L-BFGS algorithm, all the computations can be performed locally on each partition except the dot product computation which needs different computational nodes to share their information. The ECL language of the HPCC Systems platform simplifies implementing parallel computations which are done locally on each computational node, as well as performing global computations where computational nodes share information. We explained how we used these capabilities to implement L-BFGS algorithm on a HPCC platform. Our experimental results show that our implementation of the L-BFGS algorithm can scale from handling millions of parameters on dozens of machines to billions of parameters on hundreds of machines. The implemented L-BFGS algorithm can be used for parameter estimation in machine learning problems with a very large number of parameters. Additionally, It can be used in image or text classification applications, where the large number of features and classes naturally increase the number of model parameters, especially for models such as deep neural networks.

Compared to the parallelized implementation of L-BFGS called Sandblaster, by Google, the HPCC Systems implementation does not require adding any new component such as a parameter server to the framework. HPCC Systems is an open source platform which already provides the data-centric parallel computing capabilities. It can be used by practitioners to implement their large-scale models without the need to design a new framework. In future work, we want to use the HPCC Systems parallelization capabilities on each node which is done through multithreaded processing to further speed up our implementations.

\section{Authors' contributions}

MMN carried out the conception and design of the research, performed the implementations and drafted the manuscript. TMK, FV and JH provided reviews on the manuscript. JH set up the experimental framework on AWS and provided expert advice on ECL. All authors read and approved the final manuscript.

Author details

${ }^{1}$ Florida Atlantic University, 777 Glades Road, Boca Raton, FL, USA. ${ }^{2}$ LexisNexis Business Information Solutions, 245 Peachtree Center Avenue, Atlanta, GA, USA.

\section{Acknowledgements}

Not applicable.

Competing interests

The authors declare that they have no competing interests.

\section{Publisher's Note}

Springer Nature remains neutral with regard to jurisdictional claims in published maps and institutional affiliations. 


\section{References}

1. Bennett KP, Parrado-Hernández E. The interplay of optimization and machine learning research. J Mach Learn Res. 2006;7:1265-81.

2. Najafabadi MM, Villanustre F, Khoshgoftaar TM, Seliya N, Wald R, Muharemagic E. Deep learning applications and challenges in big data analytics. J Big Data. 2015;2(1):1-21.

3. Xing EP, Ho Q, Xie P, Wei D. Strategies and principles of distributed machine learning on big data. Engineering. 2016;2(2):179-95.

4. Dean J, Corrado G, Monga R, Chen K, Devin M, Mao M, Senior A, Tucker P, Yang K, Le QV, et al. Large scale distributed deep networks. In: Advances in neural information processing systems. Lake Tahoe, Nevada: Curran Associates Inc.; 2012. p. 1223-31.

5. Krizhevsky A, Sutskever I, Hinton GE. Imagenet classification with deep convolutional neural networks. In: Pereira F, Burges CJC, Bottou L, Weinberger KQ, editors. Advances in neural information processing systems. Lake Tahoe, Nevada: Curran Associates, Inc.; 2012. p. 1097-05.

6. Dong L, Lin Z, Liang Y, He L, Zhang N, Chen Q, Cao X, Izquierdo E. A hierarchical distributed processing framework for big image data. IEEE Trans Big Data. 2016;2(4):297-309.

7. Sliwinski TS, Kang SL. Applying parallel computing techniques to analyze terabyte atmospheric boundary layer model outputs. Big Data Res. 2017;7:31-41.

8. Shalev-Shwartz S, Singer Y, Srebro N. Pegasos: primal estimated sub-gradient solver for svm. In: Proceedings of the 24th international conference on machine learning. New York: ACM; 2007. p. 807-14

9. Zinkevich M, Weimer M, Li L, Smola AJ. Parallelized stochastic gradient descent. In: Lafferty JD, Williams CKI, ShaweTaylor J, Zemel RS, Culotta A, editors. Advances in neural information processing systems. Vancouver, British Columbia, Canada: Curran Associates Inc.; 2010. p. 2595-03.

10. Nocedal J, Wright SJ. Numerical optimization. 2nd ed. New York: Springer; 2006.

11. Ngiam J, Coates A, Lahiri A, Prochnow B, Le QV, Ng AY. On optimization methods for deep learning. In: Proceedings of the 28th international conference on machine learning (ICML-11). 2011. p. 265-72.

12. Schraudolph NN, Yu J, Günter S, et al. A stochastic quasi-newton method for online convex optimization. Artif Intell Stat Conf. 2007;7:436-43.

13. Daumé III, H.: Notes on cg and Im-bfgs optimization of logistic regression. http://www.umiacs.umd.edu/ hal/docs/ daume04cg-bfgs, implementation http://www.umiacs.umd.edu/ hal/megam/. 2004; 198: 282.

14. Middleton A, Solutions P. Hpcc systems: introduction to hpcc (high-performance computing cluster). White paper, LexisNexis Risk Solutions. 2011. http://cdn.hpccsystems.com/whitepapers/wp_introduction_HPCC.pdf.

15. Dean J, Ghemawat S. Mapreduce: simplified data processing on large clusters. Commun ACM. 2008;51(1):107-13.

16. White T. Hadoop: the definitive guide. 3rd ed. 2012

17. Datasets, R.D.: A faulttolerant abstraction for inmemory cluster computing Matei Zaharia, Mosharaf Chowdhury, Tathagata Das, Ankur Dave, Justin Ma, Murphy Mccauley, Michael J. Franklin, Scott Shenker, Ion Stoica University of California: Berkeley.

18. Agarwal A, Chapelle O, Dudík M, Langford J. A reliable effective terascale linear learning system. J Mach Learn Res. 2014:15(1):1111-33.

19. Sra S, Nowozin S, Wright SJ. Optimization for machine learning. Cambridge: The MIT Press; 2011.

20. Dekel O, Gilad-Bachrach R, Shamir O, Xiao L. Optimal distributed online prediction using mini-batches. J Mach Learn Res. 2012;13:165-202

21. Teo CH, Smola A, Vishwanathan S, Le QV. A scalable modular convex solver for regularized risk minimization. In: Proceedings of the 13th ACM SIGKDD international conference on knowledge discovery and data mining. New York: ACM; 2007. p. 727-36

22. Recht B, Re C, Wright S, Niu F. Hogwild: a lock-free approach to parallelizing stochastic gradient descent. In: ShaweTaylor J, Zemel RS, Bartlett PL, Pereira F, Weinberger KQ, editors. Advances in neural information processing systems. Granada, Spain: Curran Associates, Inc.; 2011. p. 693-701.

23. Dean J, Ghemawat S. Mapreduce: a flexible data processing tool. Commun ACM. 2010;53(1):72-7.

24. Chaiken R, Jenkins B, Larson P- $\AA$, Ramsey B, Shakib D, Weaver S, Zhou J. Scope: easy and efficient parallel processing of massive data sets. Proc VLDB Endow. 2008;1(2):1265-76.

25. Stonebraker M, Abadi D, DeWitt DJ, Madden S, Paulson E, Pavlo A, Rasin A. Mapreduce and parallel dbmss: friends or foes? Commun ACM. 2010;53(1):64-71.

26. Pike R, Dorward S, Griesemer R, Quinlan S. Interpreting the data: parallel analysis with sawzall. Sci Program. 2005;13(4):277-98

27. Gates AF, Natkovich O, Chopra S, Kamath P, Narayanamurthy SM, Olston C, Reed B, Srinivasan S, Srivastava U. Building a high-level dataflow system on top of map-reduce: the pig experience. Proc VLDB Endow. 2009;2(2):1414-25.

28. Abadi M, Agarwal A, Barham P, Brevdo E, Chen Z, Citro C, Corrado GS, Davis A, Dean J, Devin M, et al. Tensorflow: large-scale machine learning on heterogeneous distributed systems. arXiv preprint. 2016. arXiv:1603.04467.

29. Wolfe P. Convergence conditions for ascent methods. SIAM Rev. 1969:11(2):226-35.

30. Team BRD. Ecl language reference. White paper, LexisNexis Risk Solutions. 2015. http://cdn.hpccsystems.com/install/ docs/3_4_0_1/ECLLanguageReference.pdf.

31. Bishop CM. Pattern recognition and machine learning (information science and statistics). Secaucus: Springer; 2006

32. Schmidt M. minFunc: unconstrained differentiable multivariate optimization in Matlab. 2005. http://www.cs.ubc. $\mathrm{ca} /$ schmidtm/Software/minFunc.html. 